\title{
1/N expansion for Horava-Lifshitz like four-fermion models
}

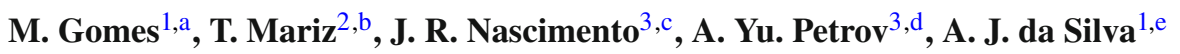 \\ ${ }^{1}$ Instituto de Física, Universidade de São Paulo, Caixa Postal 66318, São Paulo, SP 05315-970, Brazil \\ ${ }^{2}$ Instituto de Física, Universidade Federal de Alagoas, Maceió, Alagoas 57072-270, Brazil \\ ${ }^{3}$ Departamento de Física, Universidade Federal da Paraíba, Caixa Postal 5008, João Pessoa, Paraíba 58051-970, Brazil
}

Received: 2 April 2020 / Accepted: 29 May 2020 / Published online: 10 June 2020

(C) The Author(s) 2020

\begin{abstract}
We study a class of four-fermion Gross-Neveu like models in four dimensions with critical exponents $z=2$ and $z=3$. The models with $z=2$ are in general perturbatively nonrenormalizable but we show that there are in special cases renormalizable in the context of the $1 / N$ expansion. We calculate explicitly the effective potential for these models.
\end{abstract}

\section{Introduction}

Horava-Lifshitz like field theories are distinguished for the presence of higher spatial derivative terms in the Lagrangian density, while the terms involving temporal derivatives have the same form as in the usual relativistic theories [1-5]. In this way the canonical structure is preserved avoiding a possible unitarity breakdown. Asymptotically, when terms involving dimensional parameters may be neglected, these theories possess an anisotropic scale invariance with $t \rightarrow \lambda^{-z} t$ and $\mathbf{x} \rightarrow \lambda^{-1} \mathbf{x}$ where $z$, the so called dynamical critical exponent, measures the highest degree of the spatial derivatives. Because of the higher derivatives, the ultraviolet behavior of the Green functions is improved allowing the completion of theories that otherwise would be nonrenormalizable. Indeed, it has been argued that gravitation would be power counting renormalizable for $z=3$ [2]. However, because of the intrinsic Lorentz symmetry breakdown which accompanies these theories, one has to demonstrate that Lorentz invariance emerges at low energies, since, as it is well known, the Lorentz symmetry is tested experimentally with a high degree of precision for the usual energy scale, see f.e. [6]. Nevertheless, there are different gravity models which do not

\footnotetext{
a e-mail: mgomes@if.usp.br

b e-mail: tmariz@fis.ufal.br

ce-mail: jroberto@fisica.ufpb.br

de-mail: petrov@ fisica.ufpb.br (corresponding author)

e e-mail: ajsilva@if.usp.br
}

display Lorentz invariance even in the low-energy limit, see f.e. [7-10], therefore, the problem of recovering the Lorentz symmetry at the low-energy limit requires special analysis. Renormalization group methods are in general employed in such endeavors [11-13].

In this work we will study in $3+1$ dimensions a class of models with quartic self-interactions of spinor fields. In the development of field theory this class occupies a prominent position both in its conceptual aspects as well as in its applications. Thus, the Thirring or the Gross-Neveu model in two space-time dimensions introduced new concepts as anomalous dimension, fermion-antifermion bound states and Wilson short distance expansions. Above two dimensions these models are not renormalizable in the usual sense (in $2+1$ dimensions they are renormalizable only in the context of the $1 / N$ expansion). In spite of that, Nambu-JonaLasinio like models $[14,15]$ have been used in four dimensions as effective theories to investigate the chiral symmetry breakdown, with cutoffs to control the ultraviolet divergences. Horava-Lifshitz like four fermion models in $2+1$ dimensions were analyzed in $[16,17]$. Some studies in four dimensions with $z=3$ have also been realized [18]. Here we extend the three-dimensional studies performed in [16] to the four-dimensional case by considering a class of models with dynamical critical exponents $z=2$ and $z=3$. From another side, our results generalize studies performed in $[18,19]$ due to consideration of an additional coupling.

The structure of this paper looks like follows. In the section 2, we present the Horava-Lifshitz like Gross-Neveu model that we are going to study, discuss some of its symmetries and introduce auxiliary fields adequate to develop the $1 / N$ expansion; there we also perform quantum calculations to fix the effective potential for the $z=3$ case. In Sect. 3 we consider the model with $z=2$, examine the possibility of the chiral symmetry breaking and show that this is most easily accomplished by adding a term linear in the spatial derivatives to the Lagrangian density. Section 4 con- 
tains some observations on the model at finite temperature and, finally, Sect. 5 contains a Summary where our results are discussed.

\section{Horava-Lifshitz like Gross-Neveu model}

In this paper, we study the model described by the Lagrangian density

$$
\begin{aligned}
\mathcal{L}= & \bar{\psi}_{a}\left[i \gamma^{0} \partial_{0}+b_{z}\left(i \gamma^{i} \partial_{i}\right)^{z}-m^{z}\right] \psi_{a} \\
& +\frac{g_{\sigma}}{2 N}\left(\bar{\psi}_{a} \psi_{a}\right)^{2}-\frac{g_{\sigma_{5}}}{2 N}\left(\bar{\psi}_{a} \gamma_{5} \psi_{a}\right)^{2}
\end{aligned}
$$

where $\psi_{a}$ denotes a $N$-tuple, $a=1,2, \ldots N$, of fourcomponent fermion fields. As the field $\psi_{a}$ has mass dimension $3 / 2$, the self-interactions above are perturbatively nonrenormalizable if $z<3$. However, as we will shortly show, with some caveats, they are renormalizable for $z=2$ in the context of the $1 / N$ expansion and dimensional regularization in the spatial part of the integration in the internal momenta.

For $g_{\sigma_{5}}=0, m=0$ and $z=1$ or 3 , Eq. (1) describes the usual Gross-Neveu model and one of its higher spatial derivative extensions. They are invariant under the discrete transformation $\psi_{a} \rightarrow \gamma_{5} \psi_{a}$. This symmetry is explicitly broken by the mass term $m^{z} \bar{\psi}_{a} \psi_{a}$ and also by the kinetic part of the Lagrangian if $z=2$. For $g_{\sigma}=g_{\sigma_{5}}, m=0$ and $z=1$ we have the chiral Gross-Neveu model which is invariant under the chiral transformation $\psi_{a} \rightarrow e^{i \alpha \gamma_{5}} \psi_{a}$. This symmetry is also shared by the model with $z=3$ but it is broken if $m \neq 0$ or if $z=2$. Actually, the model with $z=2$ is invariant only on the anisotropic scaling.

The essence of the $1 / N$ expansion methodology [20,21] that we will employ can be explained as follows. Within this approach, one adjust the couplings of the theory so that, taking into account the dependence on $N$ of the amplitudes, one constructs a systematic $1 / N$ expansion. This is most easily accomplished by introducing auxiliary fields (scalar and pseudo-scalar, in our case) coupled to the fermions so that the quartic fermionic coupling is rewritten as a cubic coupling between the fermions and the auxiliary fields as follows

$$
\begin{gathered}
\mathcal{L}=\bar{\psi}_{a}\left[i \gamma^{0} \partial_{0}+b_{z}\left(i \gamma^{i} \partial_{i}\right)^{z}\right] \psi_{a}-\sigma \bar{\psi}_{a} \psi_{a} \\
-\frac{N}{2 g_{\sigma}} \sigma^{2}-\sigma_{5} \bar{\psi}_{a} i \gamma_{5} \psi_{a}-\frac{N}{2 g_{\sigma_{5}}} \sigma_{5}^{2},
\end{gathered}
$$

By the use of the equations of motion for the auxiliary fields $\sigma$ and $\sigma_{5}$ or by functionally integrating on them, we may reobtain Eq. (1) with $m=0$ [22]. In the $1 / N$ expansion, the propagator for the $\sigma$ field, $\Delta_{\sigma \sigma}$, receives in the tree approximation the contribution $i g_{\sigma} / N$. Besides that, in the same $1 / N$ order there is also the contribution coming from the one-loop graph, so that the complete propagator in $1 / N$ order is given by the inverse of

$\Gamma_{\sigma \sigma}(p)=-\frac{i N}{g_{\sigma}}+N \int \frac{d k_{0} d^{3} k}{(2 \pi)^{4}} \operatorname{Tr}[S(k) S(k+p)]$,

where $S(k)$ denotes the propagator for one of the fermion fields. Note that the factor $N$ in the second term comes from the fact that there are $N$ fermion flavors. In the computation of the Green functions, one uses the above propagator but oneloop graphs with two internal fermion must be discarded as they already have been incorporated into the propagator of the field $\sigma$. Similar analysis can be extended to the propagator for the $\sigma_{5}$ field.

As the propagators for the auxiliary fields carry the $1 / N$ factor and any closed fermionic loop carries the factor $N$, a contribution from an arbitrary Feynman diagram is proportional to $N^{L_{f}-n_{\sigma}}$, where $L_{f}$ is the number of fermionic loops, and $n_{\sigma}$ is the number of propagators of the auxiliary fields. Typically, one has $L_{f}-n_{\sigma} \leq-1$, except for the one-loop diagrams contributing to the fermionic determinant when the fermions are integrated out. As a result, an arbitrary Feynman diagram is proportional to $1 / N^{a}$ where $a$ is some integer $\geq-1$. This $1 / N$ expansion can be treated as an alternative to the usual loop expansion. As we will see within our calculations, the contributions to the effective potential we study are the dominant ones, proportional to $N$.

In the new form of the Lagrangian, the chiral symmetry, which holds for $g_{\sigma}=g_{\sigma_{5}}$, corresponds to the transformation $\psi \rightarrow \mathrm{e}^{i \gamma_{5} \theta} \psi$ and

$$
\left(\begin{array}{c}
\sigma \\
\sigma_{5}
\end{array}\right) \rightarrow\left(\begin{array}{cc}
\cos (2 \theta) & \sin (2 \theta) \\
-\sin (2 \theta) & \cos (2 \theta)
\end{array}\right)\left(\begin{array}{c}
\sigma \\
\sigma_{5}
\end{array}\right) \text {. }
$$

Let $\sigma_{0}$ and $\sigma_{50}$ be the vacuum expectation values (v.e.v.) of $\sigma$ and $\sigma_{5}$, respectively. If any of them is nonvanishing, the discrete symmetry $\psi_{a} \rightarrow \gamma_{5} \psi_{a}$ is explicitly broken. By shifting the fields $\sigma$ and $\sigma_{5}$ so that $\sigma \rightarrow \sigma+\sigma_{0}$ and $\sigma_{5} \rightarrow$ $\sigma_{5}+\sigma_{50}$, the Lagrangian density becomes

$$
\begin{aligned}
\mathcal{L}= & \bar{\psi}_{a}\left[i \gamma^{0} \partial_{0}+b_{z}\left(i \gamma^{i} \partial_{i}\right)^{z}\right. \\
& \left.-\sigma_{0}-i \sigma_{50} \gamma_{5}\right] \psi_{a}-\sigma \bar{\psi}_{a} \psi_{a}-\frac{N}{2 g_{\sigma}}\left(\sigma+\sigma_{0}\right)^{2} \\
& -\sigma_{5} \bar{\psi}_{a} i \gamma_{5} \psi_{a}-\frac{N}{2 g_{\sigma_{5}}}\left(\sigma_{5}+\sigma_{50}\right)^{2},
\end{aligned}
$$

furnishing the free propagator $\left(\not k \equiv k^{i} \gamma_{i}\right)$

$$
\begin{aligned}
& <\psi_{a}(k) \bar{\psi}_{b}(-k)>=S[k] \delta_{a b} \\
& =i \frac{\gamma_{0} k_{0}-i \sigma_{50} \gamma_{5}+b_{z}(\not k)^{z}+\sigma_{0}}{k_{0}^{2}-\sigma_{50}^{2}-b_{z}^{2} k^{2 z}-\sigma_{0}^{2}+i \epsilon} \delta_{a b},
\end{aligned}
$$

for $z=1$ or 3 and

$$
<\psi_{a}(k) \bar{\psi}_{b}(-k)>=S[k] \delta_{a b}=i \frac{\gamma_{0} k_{0}-i \sigma_{50} \gamma_{5}+w}{k_{0}^{2}-\sigma_{50}^{2}-w^{2}+i \epsilon} \delta_{a b},
$$


with $w=b_{2} \mathbf{k}^{2}+\sigma_{0}$, for $z=2$. Thus, for $z=1$ or 3 , the condition that the new auxiliary fields have vanishing v.e.v. gives the tadpole equations

$$
\begin{aligned}
& -i N \frac{\sigma_{0}}{g_{\sigma}}-4 N \sigma_{0} I\left[\sigma_{0}, \sigma_{50} ; z\right]=0, \\
& -i N \frac{\sigma_{50}}{g_{\sigma 5}}-4 N \sigma_{50} I\left[\sigma_{0}, \sigma_{50} ; z\right]=0,
\end{aligned}
$$

where

$$
I\left[\sigma_{0}, \sigma_{50} ; z\right]=\int \frac{d k_{0} d^{3} k}{(2 \pi)^{4}} \frac{1}{k_{0}^{2}-\sigma_{50}^{2}-b_{z}^{2} k^{2 z}-\sigma_{0}^{2}+i \epsilon} .
$$

The integral above can be easily calculated. We first integrate on $k_{0}$ and afterwards promote the remaining 3-dimensional integral to $d$ dimensions. For $z=3$ and $z=1$, respectively, employing dimensional regularization in the spatial part, we have

$$
\begin{aligned}
I\left[\sigma_{0}, \sigma_{50} ; 3\right]= & \int \frac{d k_{0} d^{d} k}{(2 \pi)^{(d+1)}} \frac{\mu^{3-d}}{k_{0}^{2}-\sigma_{50}^{2}-b_{3}^{2} k^{6}-\sigma_{0}^{2}+i \epsilon}= \\
& -\frac{i \pi}{2 b_{3}^{d / 3}} \int \frac{d^{d} k}{(2 \pi)^{d}} \frac{\mu^{3-d}}{\sqrt{k^{6}+\sigma_{0}^{2}+\sigma_{50}^{2}}} \\
= & \frac{i}{4 \pi^{2} b_{3}} \frac{1}{d-3}+\frac{i}{24 \pi^{2} b_{3}} \ln \left(\frac{\sigma_{0}^{2}+\sigma_{50}^{2}}{\mu^{6}}\right)
\end{aligned}
$$

and

$$
\begin{aligned}
I\left[\sigma_{0}, \sigma_{50} ; 1\right] & =\int \frac{d k_{0} d^{d} k}{(2 \pi)^{(d+1)}} \frac{\mu^{3-d}}{k_{0}^{2}-\sigma_{50}^{2}-b_{1}^{2} k^{2}-\sigma_{0}^{2}+i \epsilon} \\
& =-\frac{i \pi}{2 b_{1}^{d}} \int \frac{d^{d} k}{(2 \pi)^{d}} \frac{\mu^{3-d}}{\sqrt{k^{2}+\sigma_{0}^{2}+\sigma_{50}^{2}}} \\
& =\frac{-i\left(\sigma_{0}^{2}+\sigma_{50}^{2}\right)}{8 \pi^{2} b_{1}^{3}(d-3)}-\frac{i\left(\sigma_{0}^{2}+\sigma_{50}^{2}\right)}{16 \pi^{2} b_{1}^{3}} \ln \left(\frac{\sigma_{0}^{2}+\sigma_{50}^{2}}{\mu^{2}}\right),
\end{aligned}
$$

where, to simplify the final results, we have redefined the renormalization spot, above designated by $\mu$, to absorb some finite constants (terms that vanish when $d=3$ have been neglected). The divergences in the above expressions may be eliminated by conveniently defining the renormalized coupling constants. For the chiral model we adopt the same counterterm so that

$$
\begin{aligned}
& \frac{1}{g_{\sigma R}}=\frac{1}{g_{\sigma}}+\frac{1}{\pi^{2} b_{3}} \frac{1}{d-3}, \\
& \frac{1}{g_{\sigma_{5 R}}}=\frac{1}{g_{\sigma_{5}}}+\frac{1}{\pi^{2} b_{3}} \frac{1}{d-3},
\end{aligned}
$$

and therefore we can choose

$$
\frac{1}{g} \equiv \frac{1}{g_{\sigma R}}=\frac{1}{g_{\sigma_{5 R}}}=-\frac{1}{6 \pi^{2} b_{3}} \ln \left(\frac{\sigma_{0}^{2}+\sigma_{50}^{2}}{\mu^{6}}\right) .
$$

Assuming that $b_{3}$ does not depend on $\mu$, the invariance of this result under the renormalization group,

$$
\left(\mu \frac{\partial}{\partial \mu}+\beta \frac{\partial}{\partial g}\right) \rho=0,
$$

where $\rho \equiv \sqrt{\sigma_{0}^{2}+\sigma_{50}^{2}}$, fixes $\beta=-\frac{g^{2}}{\pi^{2} b_{3}}$, so that the model is asymptotically free, which agrees with [18] (for study of the renormalization group in Horava-Lifshitz theories see also [23]).

One important consequence of Eq. (8) is the cancellation of divergences in the two point vertex functions of the auxiliary fields. Indeed, up to one loop,

$$
\begin{aligned}
\Gamma_{\sigma \sigma}(p) & =-\frac{i N}{g_{\sigma}}+N \int \frac{d k_{0} d^{3} k}{(2 \pi)^{4}} \operatorname{Tr}[S(k) S(k+p)], \\
\Gamma_{\sigma_{5} \sigma_{5}}(p) & =-\frac{i N}{g_{\sigma_{5}}}-N \int \frac{d k_{0} d^{3} k}{(2 \pi)^{4}} \operatorname{Tr}\left[S(k) \gamma_{5} S(k+p) \gamma_{5}\right]
\end{aligned}
$$

and

$\Gamma_{\sigma_{5} \sigma}(p)=i N \int \frac{d k_{0} d^{3} k}{(2 \pi)^{4}} \operatorname{Tr}\left[S(k) \gamma_{5} S(k+p)\right]$.

For $z=3$ the would be (logarithmic) divergences of these expressions are in fact absent and we obtain

$$
\begin{aligned}
\Gamma_{\sigma \sigma}(0) & =-8 N \int \frac{d k_{0} d^{3} k}{(2 \pi)^{4}} \frac{\sigma_{0}^{2}}{\left(k_{0}^{2}-b_{3}^{2} k^{6}-\sigma_{50}^{2}-\sigma_{0}^{2}\right)^{2}} \\
& =-\frac{N i}{3 \pi^{2} b_{3}} \frac{\sigma_{0}^{2}}{\sigma_{0}^{2}+\sigma_{50}^{2}}, \\
\Gamma_{\sigma_{5} \sigma_{5}}(0) & =-8 N \int \frac{d k_{0} d^{3} k}{(2 \pi)^{4}} \frac{\sigma_{50}^{2}}{\left(k_{0}^{2}-b_{3}^{2} k^{6}-\sigma_{50}^{2}-\sigma_{0}^{2}\right)^{2}} \\
& =-\frac{N i}{3 \pi^{2} b_{3}} \frac{\sigma_{50}^{2}}{\sigma_{0}^{2}+\sigma_{50}^{2}}, \\
\Gamma_{\sigma_{5} \sigma}(0) & =-8 N \int \frac{d k_{0} d^{3} k}{(2 \pi)^{4}} \frac{\sigma_{0} \sigma_{50}}{\left(k_{0}^{2}-b_{3}^{2} k^{6}-\sigma_{50}^{2}-\sigma_{0}^{2}\right)^{2}} \\
& =-\frac{N i}{3 \pi^{2} b_{3}} \frac{\sigma_{0} \sigma_{50}}{\sigma_{0}^{2}+\sigma_{50}^{2}} .
\end{aligned}
$$

Similarly, for $z=1$ the highest (quadratic) divergence is cancelled but a logarithmic divergence still persists. To eliminate this remaining divergence, the bare Lagrangian should contain kinetic terms for the auxiliary fields but this can not sustain since it would turn the model indistinguishable from the Yukawa model. For this reason, in this case the model is no longer renormalizable.

The effective potential for the $z=3$ model may be obtained by integrating the one-point function for the auxiliary field $\sigma$ which gives (for the calculation of the effective potential in Horava-Lifshitz models see [24-26]) 


$$
\begin{aligned}
V\left[\sigma, \sigma_{5}\right] / N= & \frac{\sigma^{2}}{2 g_{\sigma}}-4 i \int(d \sigma) \sigma I\left[\sigma, \sigma_{5} ; 3\right]+f\left[\sigma_{5}\right] \\
= & \frac{\sigma^{2}}{2 g_{\sigma}}+\frac{1}{2 \pi^{2} b_{3}} \frac{\sigma^{2}}{d-3} \\
& +\frac{\left(\sigma^{2}+\sigma_{5}^{2}\right)}{12 \pi^{2} b_{3}} \ln \frac{\left(\sigma^{2}+\sigma_{5}^{2}\right)}{\mu^{6}} \\
& -\frac{\sigma^{2}}{12 \pi^{2} b_{3}}+f\left[\sigma_{5}\right],
\end{aligned}
$$

where the function $f\left[\sigma_{5}\right]$ is fixed by imposing that the derivative of $V$ with respect to $\sigma_{5}$ coincides with the one point function of $\sigma_{5}$. Proceeding in this way we find

$f\left[\sigma_{5}\right]=\frac{\sigma_{5}^{2}}{2 g_{\sigma 5}}+\frac{1}{2 \pi^{2} b_{3}} \frac{\sigma_{5}^{2}}{d-3}-\frac{\sigma_{5}^{2}}{12 \pi^{2} b_{3}}$,

resulting that

$$
\begin{aligned}
V\left[\sigma, \sigma_{5}\right] / N= & \frac{\sigma^{2}}{2 g_{\sigma}}+\frac{\sigma_{5}^{2}}{2 g_{\sigma 5}}+\frac{1}{2 \pi^{2} b_{3}} \frac{\sigma^{2}+\sigma_{5}^{2}}{d-3} \\
& +\frac{1}{12 \pi^{2} b_{3}}\left[\left(\sigma^{2}+\sigma_{5}^{2}\right) \ln \frac{\left(\sigma^{2}+\sigma_{5}^{2}\right)}{\mu^{6}}-\sigma^{2}-\sigma_{5}^{2}\right] .
\end{aligned}
$$

Thus, if one adopts the renormalizations (12) and (13) it follows that for $z=3$

$$
\begin{aligned}
V\left[\sigma, \sigma_{5}\right] / N= & \frac{\left(\sigma^{2}+\sigma_{5}^{2}\right)}{12 \pi^{2} b_{3}} \ln \left(\frac{\sigma^{2}+\sigma_{5}^{2}}{\sigma_{0}^{2}+\sigma_{50}^{2}}\right) \\
& -\frac{1}{12 \pi^{2} b_{3}}\left(\sigma^{2}+\sigma_{5}^{2}\right) .
\end{aligned}
$$

By computing the second order derivatives, we may check that the system (17) is obtained. The above potential is rotationally symmetric with an infinite number of vacua corresponding to the generated mass $\sqrt{\sigma_{0}^{2}+\sigma_{50}^{2}}$, as discussed after Eq. (13).

\section{The model with $z=2$}

Let us now consider the case $z=2$. Here, the term with higher spatial derivatives, $\bar{\psi}_{a}\left(i \gamma^{i} \partial_{i}\right)^{2} \psi_{a}$, breaks chiral symmetry and only the anisotropic scale invariance remains. This last symmetry is also broken if either $\sigma$ or $\sigma_{5}$ or both acquires a nonvanishing v.e.v..

Proceeding as before, let again $\sigma_{0}$ and $\sigma_{50}$ be the v.e.v. of $\sigma$ and $\sigma_{5}$, respectively. The free fermion propagator is given by Eq. (7) and the analogues of Eq. (8) are

$$
\begin{aligned}
& -i \frac{\sigma_{0}}{g_{\sigma}}+i \int \frac{d k_{0} d^{3} \mathbf{k}}{(2 \pi)^{4}} \operatorname{Tr} S(k)=-i \frac{\sigma_{0}}{g_{\sigma}} \\
& -4 \int \frac{d k_{0} d^{3} \mathbf{k}}{(2 \pi)^{4}} \frac{w}{k_{0}^{2}-\sigma_{05}^{2}-w^{2}+i \epsilon}=0, \\
& -i \frac{\sigma_{50}}{g_{\sigma 5}}+i \int \frac{d k_{0} d^{3} \mathbf{k}}{(2 \pi)^{4}} \operatorname{Tr}\left[i \gamma_{5} S(k)\right] \\
& =-i \frac{\sigma_{50}}{g_{\sigma 5}}-4 \sigma_{50} \int \frac{d k_{0} d^{3} \mathbf{k}}{(2 \pi)^{4}} \frac{1}{k_{0}^{2}-\sigma_{05}^{2}-w^{2}+i \epsilon}=0 .
\end{aligned}
$$

By integrating on $k_{0}$ we obtain

$$
\begin{aligned}
& -\frac{\sigma_{0}}{g_{\sigma}}+\frac{1}{4 \pi^{3}} \int d^{3} k \frac{w}{\left(\sigma_{05}^{2}+w^{2}\right)^{1 / 2}}=0, \\
& -\frac{\sigma_{50}}{g_{\sigma 5}}+\frac{\sigma_{50}}{4 \pi^{3}} \int d^{3} k \frac{1}{\left(\sigma_{05}^{2}+w^{2}\right)^{1 / 2}}=0 .
\end{aligned}
$$

We may now envisage various possibilities. Firstly, if $\sigma_{50}=0$ then $\sigma_{0}=0$ and reciprocally. In fact, if $\sigma_{50}=0$ the second equation in Eq. (23) is automatically satisfied whereas the integral in the first equation vanishes in the context of dimensional regularization implying that $\sigma_{0}=0$. Reciprocally, if $\sigma_{0}=0$ the first equation dimensionally regularized also implies that $\sigma_{50}=0$. One should emphasize that these results are strictly dependent on the absence of a term, $b_{1} \bar{\psi}_{a} i \gamma^{i} \partial_{i} \psi_{a}$, linear in the spatial derivatives (we shall return to this point shortly).

For general nonvanishing $\sigma_{0}$ and $\sigma_{05}$, the elliptic integrals in the system of equations (23) may not be expressed in terms of simple functions. However, by assuming that $\sigma_{50}$ is small, we may go on with our analysis by developing the integrals in (23) up to second order in $\sigma_{50}$. We have, employing dimensional regularization,

$\int d^{3} k \frac{w}{\left(\sigma_{50}^{2}+w^{2}\right)^{1 / 2}} \approx \lim _{d \rightarrow 3} \int d^{d} k\left(1-\frac{\sigma_{50}^{2}}{2 w^{2}}\right)=-\frac{\pi^{2} \sigma_{50}^{2}}{2 \sigma_{0}^{1 / 2} b_{2}^{3 / 2}}$

and

$$
\begin{aligned}
& \int d^{3} k \frac{1}{\left(\sigma_{50}^{2}+w^{2}\right)^{1 / 2}} \approx \lim _{d \rightarrow 3} \int d^{d} k\left(\frac{1}{w}-\frac{\sigma_{50}^{2}}{2 w^{3}}\right) \\
& =-\frac{2 \pi^{2} \sigma_{0}^{1 / 2}}{b_{2}^{3 / 2}}-\frac{\pi^{2} \sigma_{50}^{2}}{8 \sigma_{0}^{3 / 2} b_{2}^{3 / 2}},
\end{aligned}
$$

Using these results we may obtain the effective potential, which is given by

$V_{e f f} / N=\frac{\sigma^{2}}{2 g_{\sigma}}+\frac{\sigma_{5}^{2}}{2 g_{\sigma_{5}}}+\frac{\sigma^{1 / 2} \sigma_{5}^{2}}{4 \pi b_{2}^{3 / 2}}+\frac{\sigma_{5}^{4} \sigma^{-3 / 2}}{128 \pi b_{2}^{3 / 2}}$. 
Instead of expanding in $\sigma_{50}$, another procedure consists in introducing the term $\Delta \mathcal{L}=b_{1} \bar{\psi}_{a} i \gamma^{i} \partial_{i} \psi_{a}$ in the Lagrangian, with $b_{1}$ small so that it could be treated perturbatively as we will discuss. Assuming that $\sigma_{50}=0$, a great simplification is achieved. In zeroth order in $b_{1}$, the fermion propagator, given in Eq. (7), is

$$
\begin{aligned}
<\psi_{a}(k) \bar{\psi}_{b}(-k)> & =i \frac{\gamma^{0} k_{0}+\omega}{k_{0}^{2}-\omega^{2}+i \epsilon} \delta_{a b} \\
& =\left(\frac{i P_{+}}{k_{0}-\omega+i \epsilon}-\frac{i P_{-}}{k_{0}+\omega-i \epsilon}\right) \delta_{a b},
\end{aligned}
$$

where $\omega=b_{2} \mathbf{k}^{2}+\sigma_{0}$ and $P_{ \pm}=\frac{1 \pm \gamma_{0}}{2}$ are orthogonal projectors. Because of this form of the propagator, the analytic expression for the integrand associated to a closed fermionic loop, having at its vertices just matrices which commute with $\gamma_{0}$, may be expressed as a sum of terms which have poles either in the upper or in the lower part of the complex plane of the integration variable $k_{0}$. Of course, such expression vanishes upon integration over $k_{0}$ [23]. Thus, for example, the two point vertex functions of the auxiliary fields, adopting dimensional regularization on the spatial part of the loop integration variable, are ( $\Gamma_{\sigma \sigma_{5}}=0$ due to the just mentioned property of the fermion propagator)

$$
\begin{aligned}
\Gamma_{\sigma \sigma} & =-i \frac{N}{g_{\sigma}}+N \operatorname{Tr} \int \frac{d k_{0} d^{d} k}{(2 \pi)^{d+1}}[S(k) S(k-p)]=-i \frac{N}{g_{\sigma}} \\
\Gamma_{\sigma_{5} \sigma_{5}} & =-i \frac{N}{g_{\sigma 5}}-N \operatorname{Tr} \int \frac{d k_{0} d^{d} k}{(2 \pi)^{d+1}}\left[S(k) \gamma_{5} S(k-p) \gamma_{5}\right] \\
& =-i \frac{N}{g_{\sigma 5}}+N I\left[p_{0}, p\right]
\end{aligned}
$$

where

$$
\begin{aligned}
& I\left[p_{0}, p\right]=-2 \int \frac{d k_{0} d^{d} k}{(2 \pi)^{d+1}} \\
& \times\left[\frac{1}{\left(k_{0}-w(k)+i \epsilon\right)\left(k_{0}-p_{0}+w(k-p)-i \epsilon\right)}\right. \\
& \left.\quad+\frac{1}{\left(k_{0}+w(k)-i \epsilon\right)\left(k_{0}-p_{0}-w(k-p)+i \epsilon\right)}\right] \\
& =2 i \int \frac{d^{d} k}{(2 \pi)^{d}}\left[\frac{1}{p_{0}+w(k)+w(k-p)}+\left(p_{0} \leftrightarrow-p_{0}\right)\right] .
\end{aligned}
$$

We find that the propagator of the $\sigma$ field is trivial constant since the one-loop correction to it is zero, similarly to [23]. Notice that since we have taken $\sigma_{50}=0$ then $\sigma_{0}$ is also zero; in such situation, it may be convenient to introduce a mass parameter for the propagator of the $\psi$ field. We do it through a formal replacement $w(k) \rightarrow b_{2} \mathbf{k}^{2}+m$ in (29), where $m$ is the just mentioned parameter. By performing the above straightforward integration, we get

$$
\begin{aligned}
I\left[p_{0}, p\right]= & \frac{i}{b_{2}} \frac{\Gamma[1-d / 2]}{(4 \pi)^{d / 2}}\left[\left(\frac{p^{2}}{4}+\frac{p_{0}}{2 b_{2}}+\frac{m}{b_{2}}\right)^{d / 2-1}\right. \\
& \left.+\left(\frac{p^{2}}{4}-\frac{p_{0}}{2 b_{2}}+\frac{m}{b_{2}}\right)^{d / 2-1}\right]
\end{aligned}
$$

which for $d=3$ gives

$I\left[p_{0}, p\right]=-\frac{i}{4 \pi b_{2}}\left[\sqrt{\frac{p^{2}}{4}+\frac{p_{0}}{2 b_{2}}+\frac{m}{b_{2}}}+\sqrt{\frac{p^{2}}{4}-\frac{p_{0}}{2 b_{2}}+\frac{m}{b_{2}}}\right]$.

Thus, whereas the effective propagator for the $\sigma$ is a constant independent of momentum, the corresponding effective propagator for the $\sigma_{5}$ field for large momentum decreases as $p^{-1}$ or $1 / \sqrt{p_{0}}$. This improvement that happens in the $1 / N$ expansion is crucial to construct a renormalizable theory (it does not occur in the loop expansion). Taking into account this behavior, the degree of superficial divergence for a generic graph $\gamma$ in the models with nontrivial one-loop two point vertex functions [this includes the model with $g_{\sigma}=0$ but not the one with nonvanishing $g_{\sigma}$ in (1)] of the auxiliary field $\sigma_{5}$ whose propagator linearly decreases as the momentum grows can be calculated as follows

$\delta(\gamma)=5 L-2 n_{F}-n_{A}$,

where $L$ is the number of loops and $n_{F}$ and $n_{A}$ are the numbers of internal fermion and auxiliary lines. Using now the topological identities

$L=n_{F}+n_{A}-V+1, \quad 2 n_{F}+N_{F}=2 V, \quad 2 n_{A}+N_{A}=V$,

where $N_{F}$ and $N_{A}$ are the number of external lines associated to the fermion and auxiliary fields and $V$ is the number of vertices in $\gamma$, we obtain

$\delta(\gamma)=5-\frac{3}{2} N_{F}-2 N_{A}$.

Thus, all the divergences may be eliminated by a wave function renormalization of the $\psi$ field and renormalizations of the couplings constants and of the parameters $m$ and $b_{2}$.

Similarly, for generic $z=2,3, \ldots$, using (6), we found

$\delta(\gamma)=3+z-\frac{3}{2} N_{F}-z N_{A}$.

As the above results are valid to arbitrary $1 / N$ order, we note that all the divergences are located in the two point functions $\left(N_{F}=2\right.$ or $\left.N_{A}=2\right)$ and in the three point functions $\left(N_{F}=2\right.$ and $N_{A}=1$, i.e. $N_{\sigma}=1$ or $\left.N_{\sigma 5}=1\right)$. As before, they may be eliminated by a renormalization of the parameters of the theory and of the wave function of $\psi$. Therefore 
Fig. 1 Tadpole graph with two insertions of the vertex $b_{1} \bar{\psi} i \gamma^{i} \partial_{i} \psi$. The continuous and dashed lines stand for the sigma fields propagators for the fermion and

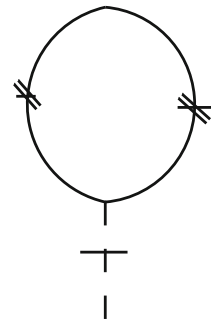

this class of theories, involving $\sigma_{5}$ field but not the $\sigma$ field is renormalizable in the $1 / N$ expansion while the theories involving the $\sigma$ field whose propagator is trivial are evidently non-renormalizable.

We still have to discuss the nonvanishing of the $\sigma$ field tadpole due to the introduction of the term linear in the spatial derivatives. In this new condition, up to second order in $b_{1}$, the tadpole equation becomes (see Fig. 1)

$$
\begin{aligned}
& -\frac{i \sigma_{0}}{g_{\sigma}}+2 b_{1}^{2} \int \frac{d k_{0} d^{3} \mathbf{k}}{(2 \pi)^{4}} \mathbf{k}^{2}\left[\frac{1}{\left(k_{0}-w+i \epsilon\right)^{2}\left(k_{0}+w-i \epsilon\right)}\right. \\
& \left.-\frac{1}{\left(k_{0}+w-i \epsilon\right)^{2}\left(k_{0}-w+i \epsilon\right)}\right]=0
\end{aligned}
$$

so that

$$
-\frac{i \sigma_{0}}{g_{\sigma}}+\frac{i b_{1}^{2}}{b_{2}^{2}} \int \frac{d^{3} k}{(2 \pi)^{3}} \frac{k^{2}}{\left(k^{2}+\frac{\sigma_{0}}{b_{2}}\right)^{2}}=0 .
$$

By using dimensional regularization, we obtain the finite result

$0=\frac{\sigma_{0}}{g_{R}}-\frac{3 b_{1}^{2}}{8 \pi b_{2}^{2}} \sqrt{\frac{\sigma_{0}}{b_{2}}}$.

This equation allows for $\sigma_{0} \neq 0$. We may also calculate the effective potential by integrating the last result. However, here we obtain it from the effective action given by

$$
\begin{aligned}
\Gamma_{e f f} & =-\int d^{4} x \frac{N \sigma^{2}}{2 g_{\sigma}}-\frac{N b_{1}^{2}}{2} \int d^{4} x \int \frac{d^{4} k}{(2 \pi)^{4}} \operatorname{Tr}[S(k) \not k S(k) \not k] \\
& =-\int d^{4} x \frac{N \sigma^{2}}{2 g_{\sigma}}-2 N \frac{b_{1}^{2}}{b_{2}^{5 / 2}} \int d^{4} x \int \frac{d k_{0} d^{3} k}{(2 \pi)^{4}} \frac{\mathbf{k}^{2}}{k_{0}^{2}+\left(\mathbf{k}^{2}+\sigma\right)^{2}},
\end{aligned}
$$

where $S[k]$ denotes the fermion propagator given in Eq. (27). Assuming $\sigma$ to be constant, disregarding a factor of volume and changing the overall sign, we obtain

$V_{e f f}=N \frac{\sigma^{2}}{2 g_{\sigma}}-N \frac{b_{1}^{2} \sigma^{3 / 2}}{4 \pi b_{2}^{5 / 2}}$.

One may then verify that its minimum is reached at $\sigma=\sigma_{0}$ satisfying Eq. (37). For positive $g_{\sigma}$, the general behavior of this effective potential is shown in Fig. 2.

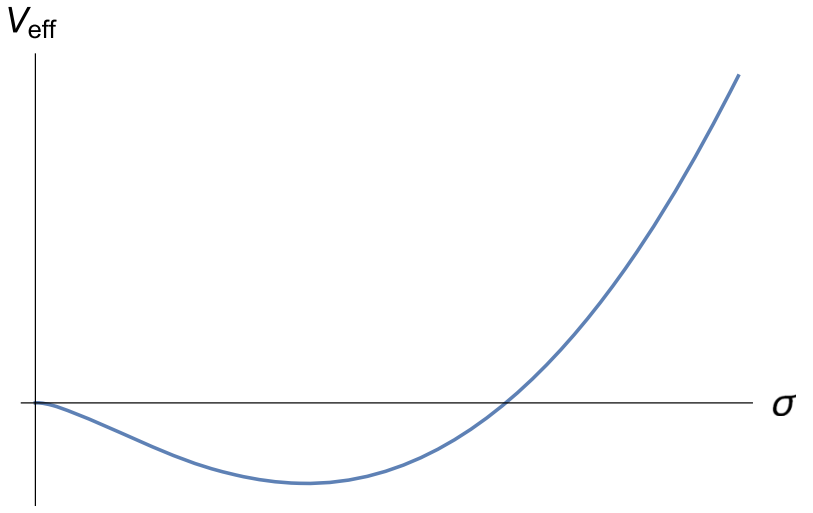

Fig. 2 General aspect of the effective potential for the one-loop effective potential in Gross-Neveu model with $z=2$

\section{Finite temperature considerations}

One can generalize the calculation of this effective potential for finite temperature. The importance of this study is motivated by the fact that finite temperature effects allow to describe phase transitions making our study more interesting from the phenomenological viewpoint. To proceed in this case, we follow the Matsubara methodology, that is, we require, in the Eq. (39), that the zeroth component of the momentum be discrete, $k_{0} \rightarrow 2 \pi T\left(n+\frac{1}{2}\right)$, with $n$ integer, and $T$ is the temperature, and the integral over $k_{0}$ been replaced by the sum over $n$. After calculating the trace, one arrives at

$$
\begin{aligned}
V_{e f f}[T]= & \frac{N \sigma^{2}}{2 g_{\sigma}}+2 N \frac{b_{1}^{2}}{b_{2}^{5 / 2}} T \\
& \sum_{n=-\infty}^{\infty} \int \frac{d^{3} \mathbf{k}}{(2 \pi)^{3}} \frac{\mathbf{k}^{2}}{4 \pi^{2} T^{2}\left(n+\frac{1}{2}\right)^{2}+\left(\mathbf{k}^{2}+\sigma\right)^{2}} .
\end{aligned}
$$

To develop this expression, it is convenient to do first the sum and afterwards the integral, as it has been done in [26]. We use the formula

$\sum_{n=-\infty}^{\infty} \frac{1}{a^{2}+\left(n+\frac{1}{2}\right)^{2}}=\pi \frac{\tanh \pi a}{a}$,

with $a^{2}=\frac{\left(\mathbf{k}^{2}+\sigma\right)^{2}}{4 \pi^{2} T^{2}}$, a dimensionless parameter. Therefore we have

$V_{e f f}[T]=\frac{N \sigma^{2}}{2 g_{\sigma}}+2 N \frac{b_{1}^{2}}{2 b_{2}^{5 / 2}} \int \frac{d^{3} \mathbf{k}}{(2 \pi)^{3}} \frac{\mathbf{k}^{2}}{\mathbf{k}^{2}+\sigma} \tanh \frac{\mathbf{k}^{2}+\sigma}{2 T}$.

We note that in the limit of zero temperature this result reproduces (39) after integration over $k_{0}$. Here we assume that the dimensional regularization is used.

It remains now to calculate the above integral. To do it, one can introduce dimensionless variables: first of all, we 
replace the integration variable $k=|\mathbf{k}|$ as $k=\sqrt{T} t$, with $t$ being a dimensionless integration variable (remind that the mass dimension of the temperature is 2 due to the anisotropic scaling), then, introduce the dimensionless parameter $\alpha^{2}=$ $\frac{\sigma}{T}$. We have

$V_{e f f}[T]=V_{e f f}(0)+\frac{T^{3 / 2}}{2 \pi^{2}} \frac{N b_{1}^{2}}{b_{2}^{5 / 2}} I$

where $V_{\text {eff }}(0)$ is the effective potential at zero temperature given by (40) and

$I=\int_{0}^{\infty} d t \frac{t^{4}}{t^{2}+\alpha^{2}}\left(\tanh \frac{t^{2}+\alpha^{2}}{2}-1\right)$.

However, the integral $I$ can be calculated only numerically, and it tends to zero at the low temperature limit $\alpha \rightarrow \infty$, as it must be, and to the constant $C_{0} \simeq-0.678904$ at the higher temperature limit $\alpha \rightarrow 0$.

\section{Summary}

Let us discuss our results. We have formulated a set of four-fermion Lifshitz-like models and showed, with use of the dimensional regularization, that they are power-counting renormalizable within $\frac{1}{N}$ expansion and dimensional regularization. For $z=2$, we obtained explicitly the two-point functions of the auxiliary fields, and for $z=3$, we verified that the renormalization of the coupling constant removes also the divergence appearing in the two-point function of the auxiliary field. We discussed two possible ways to avoid vanishing of the tadpole for the model which occurs for $z=2$ : firstly, we make a series expansion in the v.e.v. of the pseudoscalar auxiliary field, and secondly, we introduced a term linear in the derivatives of the fermion field but with the v.e.v. of the pseudoscalar field equal to zero $\left(\sigma_{50}=0\right)$. In all these cases we calculated the effective potential, and for $z=2$ we included the finite temperature counterpart. It is natural to expect that these results can be generalized for other values of the critical exponent, and that for all even $z$ the situations will be rather similar. Also, we note that the results for other spinor-scalar couplings, or, as is the same, for other fourfermion interactions, do not essentially differ. The study of the low energy limit and possible Lorentz symmetry restoration is presently in progress.

Acknowledgements The work by A. Yu. P. has been partially supported by the CNPq project no. 303783/2015-0, and the work by A. J. S. has been partially supported by the CNPq project no. 306926/2017-2.

Data Availability Statement This manuscript has no associated data or the data will not be deposited. [Authors' comment: Data sharing not applicable to this article as no datasets were generated or analysed during the current study].
Open Access This article is licensed under a Creative Commons Attribution 4.0 International License, which permits use, sharing, adaptation, distribution and reproduction in any medium or format, as long as you give appropriate credit to the original author(s) and the source, provide a link to the Creative Commons licence, and indicate if changes were made. The images or other third party material in this article are included in the article's Creative Commons licence, unless indicated otherwise in a credit line to the material. If material is not included in the article's Creative Commons licence and your intended use is not permitted by statutory regulation or exceeds the permitted use, you will need to obtain permission directly from the copyright holder. To view a copy of this licence, visit http://creativecomm ons.org/licenses/by/4.0/.

Funded by $\mathrm{SCOAP}^{3}$.

\section{References}

1. E.M. Lifshitz, Zh. Eksp. Teor. Fiz. 11, 255 \& 269 (1941)

2. P. Horava, Phys. Rev. D 79, 084008 (2009). arXiv:0901.3775

3. D. Anselmi, Ann. Phys. 324, 874 (2009). arXiv:0808.3470

4. D. Anselmi, Ann. Phys. 324, 1058 (2009). arXiv:0808.3474

5. P.R.S. Gomes, M. Gomes, Phys. Rev. D 85, 085018 (2012). arXiv: 1107.6040

6. V.A. Kostelecky, N. Russell, Rev. Mod. Phys. 83, 11 (2011). arXiv:0801.0287 [hep-ph]

7. A. Restuccia, F. Tello-Ortiz, Eur. Phys. J. C 80(2), 86 (2020). arXiv: 1908.06581 [hep-th]

8. G. Leon, A. Paliathanasis, Eur. Phys. J. C 79(9), 746 (2019). arXiv: 1902.09961 [gr-qc]

9. A. Coates, C. Melby-Thompson, S. Mukohyama, Phys. Rev. D 100, 064046 (2019). arXiv: 1805.10299 [hep-th]

10. M. Pospelov, Y. Shang, Phys. Rev D 85, 105001 (2012). arXiv: 1010.5249 [hep-th]

11. R. Iengo, J. Russo, M. Serone, JHEP 0911, 020 (2009). arXiv:0906.3477

12. J.M. Romero, J.A. Santiago, O. Gonzalez-Gaxiola, A. Zamora, Mod. Phys. Lett. A 25, 3381 (2010). arXiv:1006.0956

13. R.S. Pedro, M. Gomes, JHEP 1606, 173 (2016). arXiv: 1604.08924

14. Y. Nambu, G. Jona-Lasinio, Phys. Rev. 122, 345 (1961)

15. S.P. Klevansky, Rev. Mod. Phys. 64, 649 (1992)

16. A.M. Lima, T. Mariz, R. Martinez, J.R. Nascimento, A.Y. Petrov, R.F. Ribeiro, Phys. Rev. D 95, 065031 (2017). arXiv: 1612.05900

17. V.S. Alves, L. Fernandez, S. Lepe, L. Nascimento, F. Peña, Phys. Rev. D 97, 065015 (2018)

18. J. Alexandre, J. Brister, N. Houston, Phys. Rev. D 86, 025030 (2012). arXiv:1204.2246

19. A. Dhar, G. Mandal, S.R. Wadia, Phys. Rev. D 80, 105018 (2009). arXiv:0905.2928

20. S.R. Coleman, R. Jackiw, H.D. Politzer, Phys. Rev. D 10, 2491 (1974)

21. S. Coleman " $1 / \mathrm{N}$ ' in "Pointlike Structures Inside and Outside Hadrons", Zichichi ed. PIenum, New York (1981)

22. M. Gomes, V.O. Rivelles, A.J. da Silva, A similar analysis was done for the same model with $z=1$ in $2+1$ dimensions. Phys. Rev. D 41, 1363 (1990)

23. R. Iengo, M. Serone, Phys. Rev. D 81, 125005 (2010). arXiv: 1003.4430 [hep-th]

24. C.F. Farias, M. Gomes, J.R. Nascimento, A.Y. Petrov, A.J. da Silva, Phys. Rev. D 85, 127701 (2012). arXiv:1112.2081

25. C.F. Farias, J.R. Nascimento, A.Y. Petrov, Phys. Lett. B 719, 196 (2013). arXiv:1208.3427

26. C.F. Farias, M. Gomes, J.R. Nascimento, A.Y. Petrov, A.J. da Silva, Phys. Rev. D 89, 025014 (2014). arXiv:1311.6313 